\title{
CAR-T: trailblazing the path from clinical development to the clinic
}

\author{
Samuele Butera ${ }^{1}$
}

Received: 21 November 2017 / Revised: 21 December 2017 / Accepted: 15 January 2018 / Published online: 23 April 2018

(c) Macmillan Publishers Limited, part of Springer Nature 2018

Last year marked the beginning of a new era in cancer treatment with the historic, first regulatory approval of a chimeric antigen receptor T cell (CAR-T) therapy.

CAR-T technology is based on an elegantly, simple concept: using a patient's own immune cells to fight cancer. However, the realization of this concept was anything but simple, coming after decades of dashed hopes in the field of cell and gene therapy as scientists searched for better and safer ways to modify immune cells. It was the persistence of dedicated researchers and the courage of patients who participated in clinical trials that ultimately charted a course to success.

One of those successes took place in the labs of the University of Pennsylvania (Penn), where Dr. Carl June and his research team from the Perelman School of Medicine developed an approach that uses a disabled lentiviral vector to genetically encode $\mathrm{T}$ cells (see Fig. 1), producing CAR-T cells that are programmed to recognize and fight cancer cells. Penn published results of the CAR-T cell therapy, CTL019 (tisagenlecleucel), in three patients with leukemia, and though the findings were early, Novartis recognized the potential and made significant investments in Penn's CAR$T$ research, entering into a global collaboration with Penn in 2012 to further research, develop, and commercialize CAR$\mathrm{T}$ cell therapies, including tisagenlecleucel, for the investigational treatment of cancers.

Fast forward 5 years and we have entered a new era in cancer treatment. In August 2017, the US Food and Drug Administration (FDA) granted its first-ever approval of a CAR-T therapy and tisagenlecleucel became available for the treatment of children and young adults with B-cell precursor acute lymphoblastic leukemia (ALL) that is refractory or has relapsed at least twice.

Samuele Butera

samuele.butera@novartis.com

1 Novartis Oncology, One Health Plaza, East Hanover, NJ 079361080, USA
During those 5 years between the start of the Novartis-Penn collaboration and the FDA approval, a major effort was underway behind the scenes to bring this breakthrough to patients. What Dr. June and his colleagues had accomplished in their academic lab was significant. However, we needed to be able to replicate this process on a scale broad enough to manufacture and safely deliver a highly personalized immunocellular therapy-with each dose tailored individually to, and manufactured for, each patientfor global clinical trials and eventually commercialization.

So much innovation went into the creation of tisagenlecleucel itself, but there also had to be substantial innovation in its delivery. Unlike other therapies, for which the stages from development through commercialization are relatively siloed, the development and commercialization of tisagenlecleucel-a "living drug"-required an entirely new approach involving the seamless integration of clinical development with a pioneering manufacturing and supply chain platform.

To safely manufacture and deliver tisagenlecleucel on a global scale, we made significant investments in acquiring and establishing a state-of-the-art manufacturing facility with sufficient capacity and compliant with Current Good Manufacturing Practice (cGMP) standards, and onboarded highly trained staff (see Fig. 2). We developed a sophisticated, reproducible, reliable, and integrated manufacturing process and established a global supply chain platform that includes processes to ensure the safety, purity, potency, and identity of each patient-specific tisagenlecleucel product, including a fully compliant Chain of Identity (COI) system, which seamlessly integrates with our global manufacturing and quality systems to ensure patient safety. This GMP compliant and validated COI IT solution is crucial to the tisagenlecleucel manufacturing process, and is integrated with existing $\mathrm{COI}$ standards to maintain a rigorous $\mathrm{COI}$ from leukapheresis through manufacturing to patient infusion. To put it simply, this COI system is used so that a product manufactured for one patient doesn't go to a different patient.

We have also created an innovative structure designed to ensure safe access to tisagenlecleucel. Novartis is 


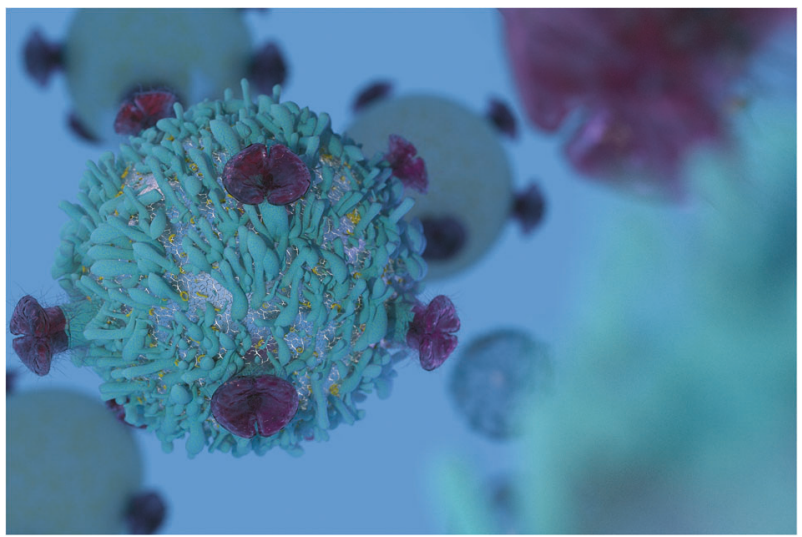

Fig. $1 \mathrm{~T}$ cell lymphocyte

establishing a network of certified treatment centers throughout the country, which will be fully trained on the use of tisagenlecleucel and appropriate patient care. All tisagenlecleucel treatment centers must be accredited by the Foundation for the Accreditation of Cellular Therapy, certified to perform allogeneic hematopoietic stem cell transplants (alloSCT), and have experience with T-cell therapies and treating leukemia. Each site is fully trained on the special ordering process, cell collection, COI, therapy use, and has received the training needed to comply with FDA requirements to support safe patient access. To serve these centers, we have a dedicated team responsible for interaction with leading physicians and nurses at the sites, as well as with the apheresis centers and emergency rooms. Further, we have established a specialized apheresis operations team comprising technical personnel who train and certify the apheresis centers to ensure the cells Novartis receives are of the highest possible quality. In addition, tisagenlecleucel is available in the US for young adult patients will B-cell ALL through a program under a Risk Evaluation and Mitigation Strategy (REMS), with the goal to mitigate the risks of cytokine release syndrome (CRS) and neurological toxicities. All hospitals and their associated clinics that administer tisagenlecleucel must be trained and certified. Novartis ensures those who prescribe, dispense, or administer tisagenlecleucel are aware of how to manage the risks of CRS and neurological toxicities.

Importantly, integration across our clinical, manufacturing, and field teams provides constant communication that allows us to continually drive optimization of quality, efficiency, and scalability.

The core of our Cell \& Gene business strategy is to now bring tisagenlecleucel and other CAR-T therapies to as many patients who can benefit as possible. In collaboration with Penn, and the Children's Hospital of Philadelphia who

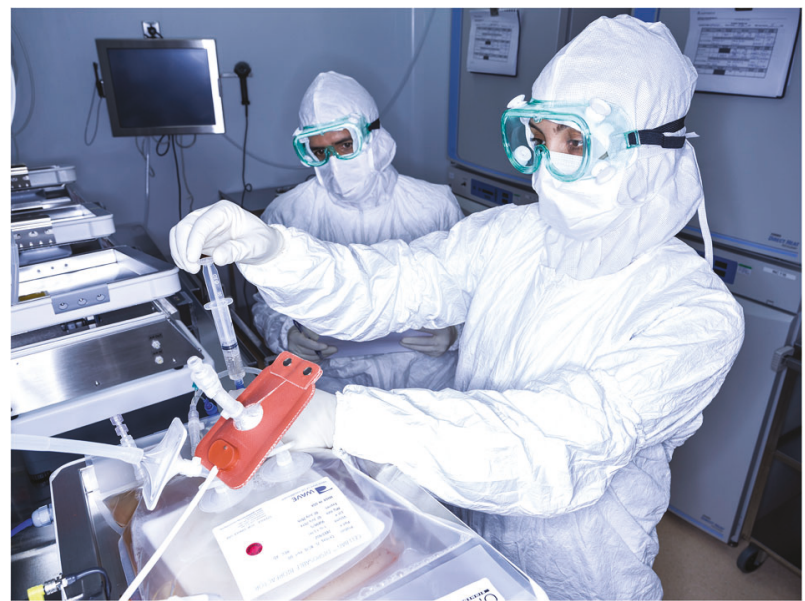

Fig. 2 Cell processing

led the first single-site trial of tisagenlecleucel in pediatric and young adult patients with $\mathrm{r} / \mathrm{r}$ B-cell ALL, we are investigating tisagenlecleucel for use in several other potentially life-threatening hematologic malignancies for which few options exist, including diffuse large B-cell lymphoma (DLBCL) and follicular lymphoma (FL). Our scientists are also studying a next-generation, humanized CD19 CAR-T in chronic lymphocytic leukemia, as well as multiple other CAR-Ts targeting antigens associated with myeloid leukemia, multiple myeloma, glioblastoma, ovarian cancer, lung cancer, and mesothelioma.

We have come a very long way, yet we and others working in this field are still only beginning to realize the potential of this technology and the ways in which we might improve it to bring even greater value to patients. Nextgeneration CAR-Ts, including bispecific antibodies and constructs which incorporate on/off switch technology, are one important path of discovery. Researchers are exploring the viability of allogeneic "off-the-shelf" CAR-Ts. We continue to study the best approach for CAR-T therapy in solid tumors, which thus far have managed to evade detection despite recent success with CAR-T in crossing the blood-brain barrier in patients with glioblastoma.

The possibilities for CAR-T therapy are still untold. What we do know is that the treatment of cancer will never be the same, and tisagenlecleucel and the first regulatory approval of a CAR-T therapy is the beginning of this new chapter.

\section{Compliance with ethical standards}

Conflict of interest The author is an employee of and receives compensation from Novartis. 